\title{
Surgical Exploration as the Definitive Means of Diagnosing a Unilateral Idiopathic Retroperitoneal Fibrosis: Report of a Case
}

\author{
Achim Troja Nader El-Sourani Dalibur Antolovic Hans-Rudolph Raab \\ Department of General and Visceral Surgery, Oldenburg Hospital gGmbH, Germany
}

\section{Keywords}

Idiopathic retroperitoneal fibrosis · Ormond's disease

\section{Summary}

Background: Retroperitoneal fibrosis is considered as one of the rare diseases, with an incidence rate of $1 / 200,000$. A guideline for its diagnosis and therapy does not exist. In the past centuries, case studies and smallscale series have therefore been used for diagnostic purposes. Case Report: We report the case of a 45-year-old female patient who approached the emergency department complaining of flank pain. The primary diagnostics including computed tomography, magnetic resonance imaging, sonography, and laboratory testing showed a distension of the Musculus psoas and Musculus iliacus on the right side with an unclear genesis. Accessorily performed diagnostic analysis including scintigraphy and intestinal endoscopy did not provide additional information. Because of persisting complaints, a surgical exploration of the right retroperitoneal space was performed resulting in a histological diagnosis of an idiopathic fibrosis (Morbus Ormond). Conclusion: Despite the permanent improvement of diagnostic imaging in general, a surgical exploration accompanied by histological investigation is the diagnostic tool of choice in selected cases.

\section{Introduction}

Retroperitoneal fibrosis is considered as one of the rare diseases, with an incidence rate of $1 / 200,000$. In the past centuries, case studies and small-scale series have been used for diagnostic purposes.
Schlüsselwörter

Idiopathische retroperitioneale Fibrose - Morbus Ormond

\section{Zusammenfassung}

Hintergrund: Die retroperitoneale Fibrose wird mit einer Inzidenzrate von 1/200 000 als eine der seltenen Erkrankungen angesehen. Eine Richtlinie für deren Diagnose und Therapie ist nicht existent. In den vergangenen Jahrhunderten wurden daher Fallberichte und kleinere Studienserien zu diagnostischen Zwecken eingesetzt. Fallbericht: Wir berichten im Folgenden über eine 45-jährige Patientin, die sich mit Flankenschmerzen in unserer Notfallambulanz vorstellte. In der bildgebenden Diagnostik samt Computertomographie, Magnetresonanztomographie, Sonographie und laborchemischen Analysen zeigte sich eine Auftreibung unklarer Genese im Musculus psoas und Musculus iliacus. Ergänzende Endoskopien und eine durchgeführte Szintigraphie führten nicht zur Diagnosestellung. Bei persistierenden Beschwerden wurde anschließend eine retroperitoneale Exploration vorgenommen. Histologisch zeigte sich das Bild einer retroperitonealen Fibrose (Morbus Ormond). Schlussfolgerung: Trotz aller Verbesserungen in der bildgebenden Diagnostik stellt die chirurgische Exploration mit anschließender Histologie zeitweise das entscheidende Diagnostikum dar.

\section{KARGER \\ Fax +497614520714}

Information@Karger.com

www.karger.com (c) 2013 S. Karger GmbH, Freiburg

$1662-6664 / 13 / 0292-0121 \$ 38.00 / 0$

Accessible online at:

www.karger.com/vim
A guideline for its diagnosis and therapy does not exist. By means of a rare case study we would like to describe the complex procedure of diagnosing retroperitoneal fibrosis. In the following case study the improvement of medical imaging is not inevitably the key in making a diagnosis. 


\section{Case Report}

\section{History}

A 45-year-old female patient presented to the surgical emergency with diffuse, right-sided abdominal pain for 1 week. On admission, she described that the symptoms had started 1 week ago in her right flank, with a progression of pain over the last few days. The past medical history included an appendectomy 15 years ago and a reactive depression without pharmacological treatment.

On the day of presentation, we performed a computed tomography (CT) of the abdomen and the pelvis showing a diffuse swelling of the right psoas muscles with adjacent reaction of the retroperitoneum.

\section{Findings}

The clinical examination upon presentation showed tenderness in the right flank, right lumbar, and right iliac fossa. There were no other clinical findings. The laboratory results showed elevated inflammatory parameters (leukocytes 11,800/ $\mathrm{ll}$; C-reactive protein $9.0 \mathrm{mg} / \mathrm{dl}$ ). All other values were within the normal range.

Considering the preliminary findings, the swelling of the psoas muscle in combination with the elevated inflammatory parameters suggested an inflammatory process. However, the CT and abdominal ultrasound could not diagnose the presence of a definitive abscess. The kidney was not involved in the inflammatory process, and the urine status did not show any abnormalities.

Further diagnostics included a magnetic resonance imaging (MRI) of the abdomen (fig. 1,2). A distinct signal uptake was seen dorsal to the right kidney. The uptake stretched from the dorsal part of the right psoas muscle and the whole iliacus muscle, the autochthone back musculature, and into the subcutaneous tissue. The spinal cord, however, was normal. A definite focus could not be identified.

\section{Therapy and Follow-up}

Regarding the elevated inflammatory parameters in combination with the inflammatory changes of the right psoas and iliacus muscle seen in the imaging process, we started an antibiotic treatment with a third-generation cephalosporin and metronidazole due to the assumption of an underlying bacterial infection. Consultations in the department of urology and gynecology were without relevant findings. A bone scintigraphy was performed to exclude any ongoing inflammatory process of the spine. In order to eliminate an intestinal dignity, a colonoscopy as well as a MR Sellink was performed. Both investigations did not show any evidence for the above-described changes or a link to the inflammatory process.

The inflammatory parameters regressed under the antibiotic therapy; however, the subjective clinical signs persisted. Due to the unsatisfactory situation, a second MRI of the abdomen and pelvis was performed 14 days after antibiotic treatment and showed no relevant new findings. Since no focus could be identified, an open biopsy via a retroperitoneal approach was indicated. As no tumor was identified in the imaging procedures (CT and MRI), we decided to perform a small retroperitoneoscopy rather than a CT-guided probe.

Intraoperatively, we found a diffuse swelling of the muscle whereas the adjacent tissue was rather sclerotic/fibrotic. The histopathological assessment described an idiopathic retroperitoneal fibrosis, i.e. Ormond's disease. There was no sign of a malignant process. The postoperative period was without any complications. The patient was discharged a few days later for an outpatient follow-up.

\section{Discussion}

Although idiopathic retroperitoneal fibrosis was first described in 1905 by Albarran and was identified by J.K. Or-
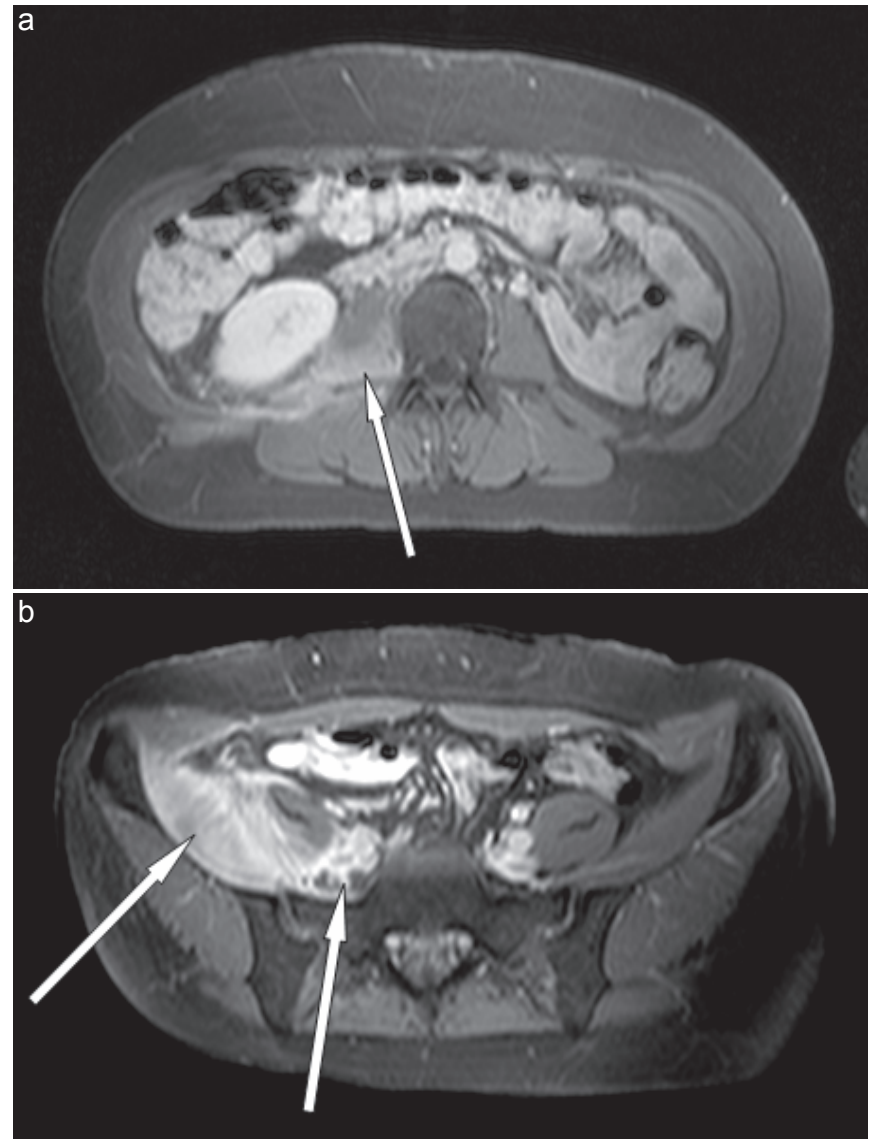

Fig. 1. a, b. MRI of the abdomen. Fibrosis is marked with white arrows.

mond as its own disease entity four decades later, Ormond's disease is considered a rare disease with an incidence rate of $1 / 200,000[1,2]$. In 1948, Ormond first described two cases in which an inflammatory retroperitoneal mass was found in the presence of a ureter obstruction. In the following years, Ormond collected data of similar cases of unidentified ureter obstructions. He divided his collective data into two groups. The data of the first group described a symmetrical fibrosis whereas the second group described a localized process [3].

Retroperitoneal fibrosis is, together with the above-mentioned incidence, a rare disease entity of the retroperitoneal fat where etiology and manifestation are vast. Principally, one divides between primary, idiopathic (Ormond's disease), and secondary retroperitoneal fibrosis.

The secondary type can be caused by radiation, infections, or pharmaceuticals (i.e. Methysegrid). The median age lies between the 5 th and 7 th decade with a male to female ratio of $3: 1$. Whereas in the past patients were diagnosed in an advanced state, with symptoms such as azotemia and symmetrical urinary retention, nowadays an early diagnosis can be made by modern imaging methods [4].

In up to $10 \%$ of the patients, retroperitoneal fibrosis is associated with an aortic aneurysm, and in $4-8 \%$ with a paraneoplastic syndrome. It is crucial to be aware of those coherences 


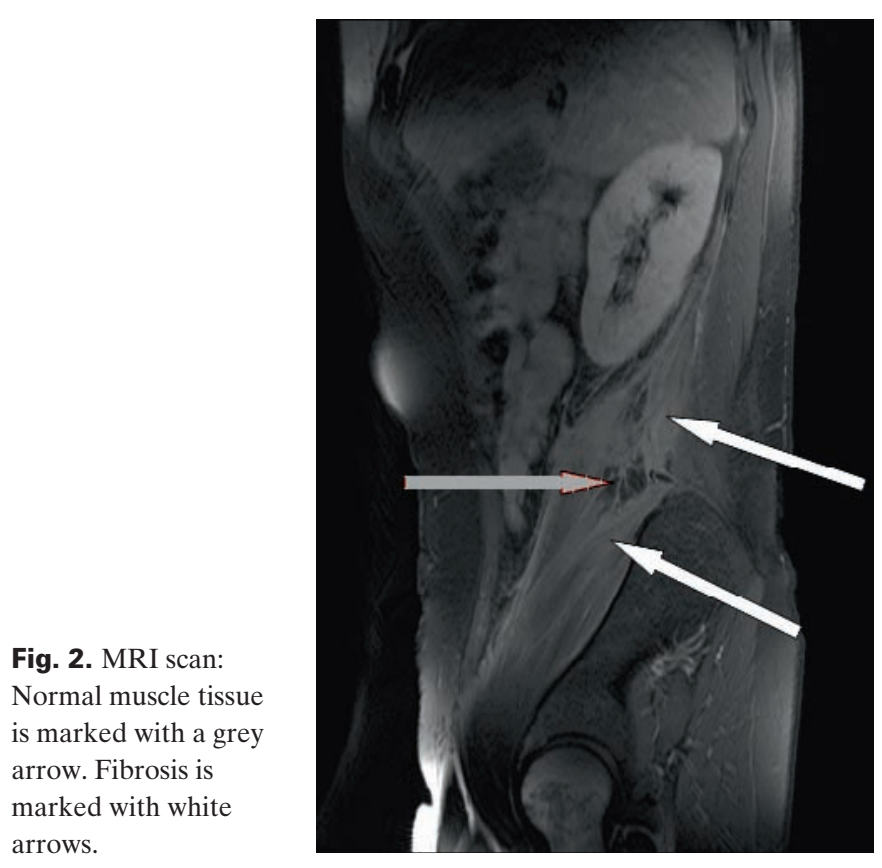

since an antibiotic therapy should only be started once the diagnostic investigations have been finished [5-7]. Up to $15 \%$ of the patients present with a process extending the borders of the retroperitoneum, which complicates diagnosing Ormond's disease; hence, a primary tumor must be considered as one of the differential diagnoses [8].

Our patient presented with right flank pain, which is described as the leading symptom in up to $40 \%$ of the cases in the available literature. In addition, back pain, polyuria, nausea, and weight loss are common symptoms [9].

In the CT, the fibrosis is often shown as plaque with the same density as the muscle rather than a diffuse swelling as described in our case. Furthermore, the performed MRI did not show the typical features of a retroperitoneal fibrosis which is distributed along the aorta, vena cava inferior, and both ureters $[10,11]$. On the contrary, the MRI described an area of intensification dorsal to the kidney without involving the ureters. Due to this we decided to pursue an open small retroperitoneoscopical approach without relevant risks to obtain a macroscopic impression of the area. It can be discussed if a CT-guided puncture might be a better solution for getting a histological probe.

The results of the clinical examination and of the imaging methods did not show a clear indication for the presence of a retroperitoneal fibrosis. Therefore, we concluded that the surgical exploration was the definitive means in diagnosing retroperitoneal fibrosis by its histopathological and macroscopical results.
Differential diagnosis such as a neoplastic disease should be considered, although lymphomas do not usually present periurethrally. Amyloidosis should also be considered as a differential diagnosis. Imaging can be applied for evaluating differential diagnosis; however, differentiating between malignant and benign entities needs to be assessed by a pathologist. A percutaneous puncture can be an alternative to surgery. We refrained from this method as the surgical exploration benefits from additional information in regard to the macroscopic picture.

Although there are no current guidelines for treating idiopathic retroperitoneal fibrosis, we used an empirically tested pharmaceutical immunosuppression therapy. Corticosteroids and/or tamoxifen are the most commonly used agents, followed by methotrexate $[12,13]$. Urological monitoring is vital in the follow-up as the fibrosis can be progressive, leading to required surgery with ureterolysis and intraperitonealization of both ureters. In this context, the above-mentioned association with malignant transformation as a paraneoplastic syndrome should be recognized. The finding of a retroperitoneal fibrosis should not exclude the possibility of an underlying malignant disease. A gastroscopy, colonoscopy, abdominal ultrasound, CT of the abdomen and pelvis, chest $\mathrm{x}$-ray as well as a urological and gynecological consultation should be performed to complete a full investigation, as done in our case study.

\section{Conclusion}

On the whole, our case study shows that the surgical exploration and the subsequent histopathological assessment are crucial for diagnosing idiopathic retroperitoneal fibrosis, especially in cases in which non-invasive investigations do not show distinct findings. Nowadays, surgical exploration can be performed via minimally invasive approaches, thereby reducing the surgical trauma to a minimum. The importance of diagnosis and knowledge of the disease, especially its association with malignancy, is crucial in order to offer the right treatment option.

This case study demonstrates that the surgical exploration, the intraoperative macroscopic picture, and the histopathological assessment were the key for diagnosing idiopathic retroperitoneal fibrosis. This led to the successful treatment with immunosuppressive agents, resulting in an arrest of disease progression by $80-90 \%[12,13]$.

\section{Disclosure Statement}

Achim Troja, Nader El-Sourani, Dalibur Antolovic and HansRudolph Raab have no conflict of interest. 


\section{References}

1 Albarran JJ: Rétention rénale par périurérite: libération externe de l'uretère. Ass Fr Urol 1905;9: 511-515.

2 Ormond JK: Bilateral ureteral obstruction due to envelopment and compression by inflammatory retroperitoneal process. J Urol 1948;59:1072-1079.

3 Ormond JK: Idiopathic retroperitoneal fibrosis, an established clinical entity. JAMA 1960;174:15611568

4 Heidenreich A, Derakhshani P, Neubauer S, Krug $\mathrm{B}$ : Behandlungsergebnisse der primären und sekundären retroperitonealen Fibrose. Urologe 2000; 39:141-148.

5 Koep L, Zuidema GD: The clinical significance of retroperitoneal fibrosis. Surg 1977;81:250-257.
6 Lepor H, Walsh PC: Idiopathic retroperitoneal fibrosis. J Urol 1979;122:1-6.

7 Parums DV, Choudhury RP, Shields SA, Davies $\mathrm{AH}$ : Characterisation of inflammatory cells associated with idiopathic retroperitoneal fibrosis. $\mathrm{Br} \mathrm{J}$ Urol 1991;67:564-568.

8 Amis ES: Retroperitoneal fibrosis. AJR Am J Roentgenol 1982;157:45-48.

9 Baker LRI, Mallinson WJW, Gregory MC, et al.: Idiopathic retroperitoneal fibrosis. A retrospective analysis of 60 cases. Br J Urol 1988;60:497-503.

10 Bachmann G, Bauer T, Rau WS: MRT und CT in Diagnose und Verlaufskontrolle der idiopathischen retroperitonealen Fibrose. Radiologie 1955; 35:200-207.
11 Kottra JJ, Dunnick NR: Retroperitoneal fibrosis Radiol Clin North Am 1996;34:1259-1275.

12 Kermani T, Crowson CS, Achenbach SJ, Luthra HS: Idiopathic retroperitoneal fibrosis: a retrospective review of clinical presentation, treatment, and outcomes. Mayo Clin Proc 2011;86:297-303.

13 Swartz RD: Idiopathic retroperitoneal fibrosis: a review of the pathogenesis and approaches to treatment. Am J Kidney Dis 2009;54:546-553. 